\title{
COMENTARIOS
}

\section{Guía de identificación de fauna silvestre, para las autoridades ambientales de Amazonas, San Martín, Loreto y Ucayali. Revisión y comentarios sobre su importancia}

\section{Guía de identificación de fauna silvestre, para las autoridades ambientales de Amazonas, San Martín, Loreto y Ucayali. A review and notes about its importance}

\section{José Eduardo Serrano-Villavicencio ${ }^{1,2 *}$, Orlando Zegarra Mori ${ }^{3}$, Dennisse Ruelas ${ }^{4}$, Marisel Flores-Quispe ${ }^{5}$, Jessica Amanzo ${ }^{6}$, Maggie Noblecilla ${ }^{4}$, Víctor Pacheco ${ }^{4}$}

1 Pós-graduação, Mastozoologia, Museu de Zoologia, Universidade de São Paulo, Avenida Nazaré, 481, CEP 04263-000, Ipiranga, São Paulo, SP, Brazil

2 Centro de Investigación Biodiversidad Sostenible (BioS), Calle Francisco de Zela 1556, Lince - Lima. Perú

3 Universidad Nacional Agraria La Molina. Av. La molina s/n, Lima 12, Perú.

4 Universidad Nacional Mayor de San Marcos, Museo de Historia Natural. Av. Arenales 1256, Lima 11, Perú

5 Universidad Latinoamericana CIMA, Av. Gregorio Albarracín 500, Tacna 23, Perú

6 IUCN/SSC Bear Specialist Group y IUCN/SSC Tapir Specialist Group University Hall, Room 309, Missoula, MT 59812, United States.

${ }^{*}$ Autor para correspondencia

Email José Serrano-Villavicencio: serranovillavicencio@gmail.com

Email Orlando Zegarra Mori: orzegarra@gmail.com

Email Dennisse Ruelas: dennisse.ruelas@unmsm.edu.pe

Email Marisel Flores-Quispe: fq.marisel@gmail.com

Email Jessica Amanzo: jessica.amanzo@gmail.com

Email Maggie Noblecilla: mcnoblecilla@gmail.com

Email Víctor Pacheco: vpachecot@unmsm.edu.pe

\section{Resumen}

Con la finalidad de brindar una herramienta para autoridades vinculadas a la lucha contra el tráfico ilegal de fauna silvestre se publicó la Guía de identificación de fauna silvestre para las autoridades ambientales de Amazonas, San Martín, Loreto y Ucayali (ISBN: 978-612-4261-24-4). Esta guía representa una herramienta importante por lo cual la información transmitida debe ser lo más actual y fidedigna posible. Sin embargo, después de revisar la sección dedicada a mamíferos, identificamos diversos errores que podrían generar confusión y ser contraproducentes en la lucha contra el tráfico ilegal de vida silvestre. Aunque resaltamos la importancia de este trabajo, sugerimos precaución en la elaboración de documentos como esta guía con la finalidad de maximizar su utilidad para las autoridades que combaten tráfico de fauna en nuestro territorio.

Palabras clave: fauna silvestre; guías de identificación; mamíferos; fauna amenazada; Amazonia; gestión ambiental; biodiversidad.

\section{Abstract}

The Guía de identificación de fauna silvestre para las autoridades ambientales de Amazonas, San Martín, Loreto y Ucayali (ISBN: 978-612-4261-24-4) was published in order to provide a tool for authorities related to the fight against wildlife trafficking and trade. This guide represents an important tool, so the information transmitted should be as current and reliable as possible. However, after reviewing the section dedicated to mammals, we identified several errors that could generate confusion and be counterproductive in the fight against wildlife trafficking. Although we emphasize the importance of this work, we suggest caution in the preparation of documents such as this guide in order to maximize its usefulness for the authorities that fight wildlife trafficking in our territory.

Keywords: wild fauna; identification guides; mammals; threatened wildlife; Amazon; environmental management; biodiversity.

Información sobre los autores:

Los autores no incurren en conflictos de intereses.

Presentado: $\quad 08 / 06 / 2018$

$16 / 06 / 2018$

Publicado online: 25/09/2018
Citación:

Serrano-Villavicencio J.E., O. Zegarra Mori, D. Ruelas, M. Flores-Quispe, J. Amanzo, M. Noblecilla, V. Pacheco. 2018. Sobre una herramienta imperfecta para la conservación de la vida silvestre que necesita ser mejorada: la Guía de identificación de fauna silvestre, para las autoridades ambientales de Amazonas, San Martín, Loreto y Ucayali. Revista peruana de biología 25(3): 349 - 354 (Agosto 2018). doi: http://dx.doi. org/10.15381/rpb.v25i3.15227

Journal home page: http://revistasinvestigacion.unmsm.edu.pe/index.php/rpb/index

(C) Los autores. Este artículo es publicado por la Revista Peruana de Biología de la Facultad de Ciencias Biológicas, Universidad Nacional Mayor de San Marcos. Este es un artículo de acceso abierto, distribuido bajo los términos de la Licencia Creative Commons Atribución-NoComercial-Compartirlgual 4.0 Internacional.(http://creativecommons.org/licenses/by-nc-sa/4.0/), que permite el uso no comercial, distribución y reproducción en cualquier medio, siempre que la obra original sea debidamente citadas. Para uso comercial, por favor póngase en contacto con editor.revperubiol@gmail.com. 


\section{Introducción}

Allgas et al. (2017) presentan la Guia de identificación de fauna silvestre para las autoridades ambientales de Amazonas, San Martin, Loreto y Ucayali (ISBN: 978-612-4261-24-4, Fig. 1) para la identificación de diversas especies peruanas de mamíferos, aves y reptiles que son objeto de tráfico ilegal. Según los autores, esta guía tiene como público objetivo a las autoridades ambientales de los departamentos de Amazonas, San Martín, Loreto y Ucayali para servir como herramienta de apoyo en materia de identificación de fauna silvestre amenazada durante las intervenciones contra el tráfico ilegal de fauna silvestre, en inspecciones de zoocriaderos, centros de rescate y centros de conservación. Los autores presentan 159 taxones que incluyen 82 especies de mamíferos, 52 de aves y 25 de reptiles.

En las primeras cuatro secciones de la guía los autores nos presentan una visión amplia del marco legal peruano, explicando de forma detallada y didáctica el proceso de denuncia penal, el código penal peruano, las categorías de clasificación de amenaza (la Lista Roja de la Unión Internacional para la Conservación de la Naturaleza, UICN y el D.S. Nº04-2014-MINAGRI), convenios de tráfico internacional de especies (Convenio sobre el Comercio Internacional de Fauna y Flora Silvestre, CITES), políticas peruanas que norman las acciones sobre fauna silvestre (Decreto Supremo No 004-2014-MINAGRI, Decreto Supremo No 019-2015-MINAGRI), entre otros procedimientos relevantes para las autoridades ambientales. Aunque la parte política y jurídica es extensamente desarrollada, no es hasta la sección "Manual de uso de la guía" en donde se detalla la estructura de las fichas de las especies en las cuales se considera la información taxonómica, las categorías de amenazas (tanto nacionales como internacionales), forma de tráfico, descripción, fotografía de la especie y mapas de distribución.

Considerando la especialidad de los autores del presente trabajo, se realizó una revisión crítica a la sección de mamíferos

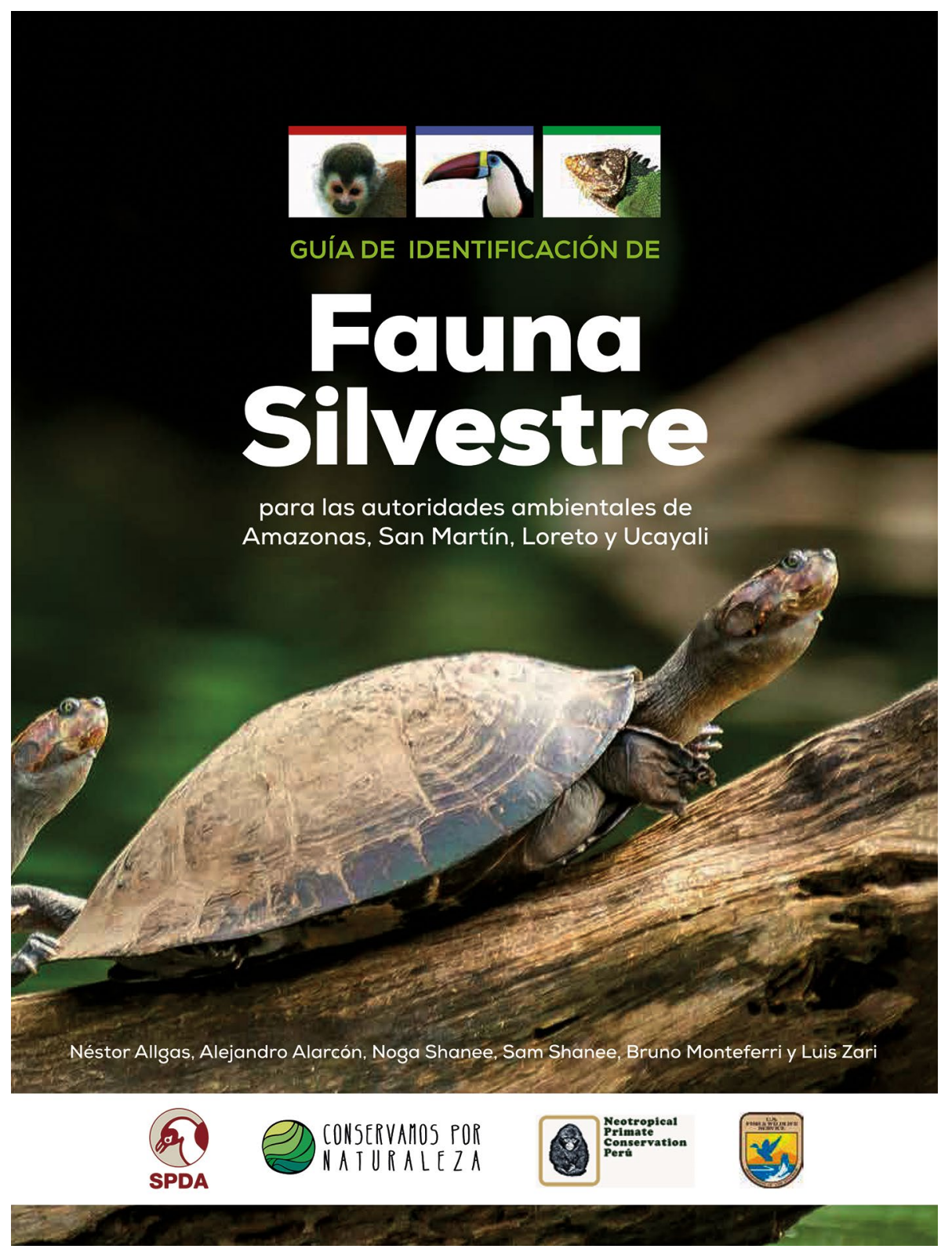

Figura 1. Caratula de la Guía de identificación de fauna silvestre para las autoridades ambientales de Amazonas, San Martín, Loreto y Ucayali (ISBN: 978-612-4261-24-4) 
(Orden Mammalia, pp. 43 - 84) con el objetivo de identificar los principales problemas que podrían conllevar a una determinación errónea de mamíferos silvestres protegidos por el Estado. Asimismo, analizamos las repercusiones legales del manejo de información inadecuada y errónea.

\section{Problemas de identidad taxonómica}

Uno de los problemas de la guía es el empleo errado de la nomenclatura. Los autores tratan como órdenes diferentes a "Artiodactila" (sic), Cetacea y Cetartiodactyla. Aunque el uso de Artiodactyla y Cetacea versus Cetartiodactyla ha sido largamente discutido por diferentes autores (e.g. Price et al. 2005, Spaulding et al. 2009, Asher \& Helgen 2010), el tratamiento dado por Allgas et al. (2017) es incorrecto, ya que Cetartiodactyla es un agrupamiento que incluye a Artiodactyla y Cetacea; por lo cual, podrían ser considerados estos dos últimos ordenes, pero bajo ninguna circunstancia las tres alternativas al mismo tiempo.

En relación al nivel específico, la guía sigue la lista de especies propuesta por la UICN, la cual genera confusión principalmente en la sección de primates, que es uno de los grupos que ha sufrido importantes cambios taxonómicos en las últimas décadas, tanto a nivel genérico (e.g. Lynch-Alfaro et al. 2012, Buckner et al. 2015; Byrne et al. 2016) como a nivel específico (e.g. van Roosmalen et al. 2002, Marsh 2014). En este caso, los autores incluyen el nombre científico de acuerdo con la UICN y entre paréntesis otro nombre actualizado con la finalidad de evitar problemas de nomenclatura, argumentando que estos nuevos nombres aún no han sido adoptados en Perú. Sin embargo, por definición, el nombre científico es único y facilita la comunicación entre investigadores debido a su carácter universal (Blackwelder 1967). Por este motivo, lo más recomendable sería escoger un solo nombre científico y, en un acápite de sinónimos, información relevante para las autoridades pertinentes.

Un ejemplo de como la guía podría generar confusión con respecto a la identificación de especies se da en la ficha de Saguinus fuscicollis. Allgas et al. (2017) informan que esta especie actualmente está "separada en varias especies" y las presentes en Perú serían Leontocebus lagonotus, Leontocebus illigeri, Leontocebus leucogenys, Leontocebus fuscicollis y Leontocebus nigrifrons, siendo todas estas morfológicamente similares. Sin embargo, no especifican a cuál de estas especies se refieren además de utilizar Saguinus fuscicollis como nombre científico válido y Leontocebus fuscicollis en otra parte de la ficha (ver página 55). Adicionalmente, los autores no presentan ningún mapa de distribución o característica de diagnóstico para cada una de ellas. Los cambios en la taxonomía de Saguinus comenzaron con Matauschek et al. (2011) que basados en evidencia molecular elevaron al nivel de especie las subespecies de $S$. fuscicollis previamente reconocidas por Hershkovitz (1977) y Groves (2001). Adicionalmente, Buckner et al. (2015) y Rylands et al. (2016) propusieron la separación del grupo monofilético Saguinus en dos géneros: Saguinus (sensu stricto) y Leontocebus. La elección de determinado arreglo taxonómico queda a criterio de los autores, siempre y cuando este sea consistente a lo largo del trabajo y se sustente explícitamente el motivo de dicha elección. Esta información no se encuentra disponible para esta sección.

\section{Problemas con ilustraciones}

El problema más preocupante de esta guía es el uso equívoco de imágenes, varias de ellas tomadas de internet sin localidad conocida y, en algunos casos, correspondientes a especies que no se encuentran en Perú. En el caso de Pudu mephistophiles se presenta una foto de Pudu puda (https://www.biolib.cz/en/ image/id11795/) la cual es una especie morfológicamente diferente y además distribuida en el extremo sur de Chile. Pudu mephistophiles se caracteriza principalmente por tener el rostro y las patas negruzcas además de la coloración lateral del cuerpo rojiza (Loyola et al. 2010), lo cual difiere notoriamente de los caracteres de diagnóstico presentados.

Un segundo ejemplo, es el caso de Mustela africana, ya que Allgas et al. (2017) presentan en la ficha de esta especie una foto en vida silvestre de Mustela lutreolina tomada de internet (http://www.gomezdefrancisco.es/images/20764/4849/) basando su descripción en ésta, que es además una especie presente en Indonesia en el continente asiático. Mustela africana es una especie considerada extremadamente rara y, hasta donde conocemos, sin registro fotográfico en estado silvestre. Esta especie es representada únicamente por algunas muestras dispersas en museos internacionales (Ramírez- Chaves et al. 2014).

Algunos otros casos con imágenes erróneas son el de Lagothrix lagothricha lagothricha (con foto de Lagothrix lagothricha lugens, subespecie presente en Colombia), Saimiri boliviensis (con foto de Saimiri oerstedii, especie presente en América Central), Odocoileus virginianus (con foto de un espécimen de Estados Unidos y no de la subespecie O.v. peruvianus), Dasyprocta kalinowskii (con foto de un Dasyprocta punctata), entre otros ejemplos.

Por otra parte, la guía cuenta con fotos en estado silvestre y en cautiverio de todas las especies con excepción de Cuniculus taczanowskii, en la cual se presenta una ilustración en blanco y negro que no permite identificar los caracteres diagnósticos relacionados al color mencionado por los autores. Otro problema de esta guía es la falta de imágenes con buena resolución que permitan identificar los caracteres diagnósticos mencionados por los autores.

\section{Problemas con características diagnósticas}

Otro punto crítico de esta guía son las descripciones de las especies, ya que estas no son precisas y pueden llevar a problemas de identificación. Un ejemplo de ello es el caso de Callicebus lucifer, una especie caracterizada por su coloración corporal negra-rojiza; sin embargo, Allgas et al. (2017) afirman que la coloración del pelaje corporal es gris oliváceo tornándose más oscuro hacia el rostro, lo cual contradice también a la imagen presentada para esta especie. En el caso de Lycalopex culpaeus, se menciona como una de las características de esta especie que la parte dorsal de la cabeza es grisácea, lo cual es erróneo, ya que de acuerdo con Novaro (1997) la característica diagnóstica de $L$. culpaeus es la coloración rojiza de la cabeza, la nuca, las orejas y las piernas. Muchas de las descripciones de otras especies omiten características relevantes para una correcta identificación ya que los caracteres presentados son muy generales. Este problema ocurre, por ejemplo, en las especies del género Leopardus. En la descripción de Leopardus pardalis mencionan como una de las características diagnósticas las manchas de los hombros, que es justamente una de las características más variables en esta especie (Murray \& Gardner 1997). En el caso de Leopardus wiedii, 
Allgas et al. (2017) mencionan que el cuerpo posee manchas moteadas bien definidas, sin embargo, de Oliveira (1998) menciona que generalmente las manchas son puntos negros y largos en la espalda media con rosetas largas y completas en los lados.

Por otra parte, Allgas et al. (2017) mencionan que la coloración del cuerpo de Puma yagouaroundi varía entre gris oscuro a negro; no obstante, según de Oliveira (1998) la coloración del pelaje es polimórfica siendo los fenotipos más comunes el oscuro (negro-pardusco) y el rojizo (rojizo-amarillento). Inclusive ambos fenotipos pueden ser encontrados en una misma camada (Cabrera \& Yepes 1960). Tampoco se menciona entre las características externas importantes de esta especie que la coloración del cuerpo es uniforme mientras que la cabeza y la región ventral del cuello pueden ser ligeramente más claras (de Oliveira 1998).

También notamos que los rangos de peso corporal y dimensiones externas contienen información inexacta. Uno de estos casos es Pteronura brasiliensis en donde se menciona que su peso corporal fluctúa entre 24 y $29 \mathrm{~kg}$, sin diferenciar los rangos entre sexos a pesar del dimorfismo sexual en el peso que presenta la especie. Según Duplaix (1980), el rango de peso de las hembras varía entre 22 a $26 \mathrm{~kg}$, mientras que el de los machos entre 26 a $32 \mathrm{~kg}$. Otro caso es el de Mustela frenata en donde se menciona que el tamaño varía entre 35 y $45 \mathrm{~cm}$; sin embargo, el rango dado por Sheffield y Thomas (1997) va de 30 a $55 \mathrm{~cm}$, además de presentar dimorfismo sexual en el tamaño, donde los machos son alrededor del doble que las hembras (Tirira 2007).

\section{Sobre las distribuciones geográficas}

Encontramos errores en los mapas de distribución en varias especies. Un ejemplo es el caso de Pithecia monachus. Hershkovitz (1987) y Marsh (2014) revisaron el género Pithecia y en ambas revisiones $P$. monachus fue reconocida como válida, aunque con diferentes perspectivas. Sin embargo, Allgas et al. (2017) presentan un mapa de distribución de Pithecia monachus que no concuerda con ninguno de los dos autores mencionados, por lo que desconocemos cuál fue la fuente de esa información. Por otra parte, el mapa de distribución presentado para Odocoileus virginianus merece una revisión mayor, ya que parece ignorar los reportes de Rheingantz et al. (2014) y Hernández-Romero et al. (2015), disminuyendo su distribución en la región norte del Perú. Por otro lado, en la distribución presentada para Dasyprocta variegata los autores no consideran la región de Chanchamayo (Junín), que es la localidad tipo de esta especie (Tschudi 1844, Gilbert 2016). Adicionalmente, Allgas et al. (2017) no presentaron ningún mapa de distribución de $P$. mephistophiles, a pesar de que esta información e imágenes de esta especie en vida silvestre están disponibles en Loyola et al. (2010).

\section{Sustento bibliográfico}

La guía presentada por Allgas et al. (2017) contiene información que resulta cuestionable, contradictoria, errónea y marcadamente insuficiente. Allgas et al. (2017) incluyen solo once referencias para toda la guía; de ellas, sólo tres son referidas a mamíferos y dos son de algunos de los autores de la guía. La tercera cita corresponde a Pacheco et al. (2009 [no 1995]) que no es usada en el texto. Si bien existen especies con poca literatura y material gráfico disponible, este no es el caso para mamíferos donde se ha generado numerosa literatura especializada y de divulgación en los últimos años y décadas, que lamentablemente los autores de la guía han soslayado (e.g. Wilson \& Reeder 2005, Wilson \& Mittermeier 2009, Mittermeier et al. 2013).

\section{Implicancias legales y para la gestión}

La problemática del tráfico ilegal de fauna silvestre en el Perú es una de las amenazas más importantes, además de ser una de las menos atendidas, para las especies de mamíferos medianos y grandes, especialmente para aquellos listados en alguna categoría de amenaza. Una de las bases necesarias para el adecuado control y fiscalización del tráfico ilegal de fauna silvestre es la correcta identificación de las especies. El uso como herramienta de una guía con información errónea dificulta aún más la compleja problemática del tráfico ilegal de fauna silvestre en el Perú, que actualmente se ubica entre los cuatro comercios ilegales más lucrativos a nivel mundial después del narcotráfico, el tráfico de personas y el de productos falsificados (Myburgh 2011). Se ha estimado que el tráfico ilegal de fauna silvestre recauda anualmente entre 5 a 23 billones de dólares a nivel mundial (May 2017). En este sentido, la Estrategia Nacional para Reducir el Tráfico Ilegal de Fauna Silvestre en el Perú 2017 - 2027 y su Plan de Acción 2017 2022 elaborado con las contribuciones de diversas instituciones de forma participativa e integradora muestra como destino final del tráfico ilegal de fauna silvestre a las colecciones particulares, zoológicos, laboratorios con fines científicos o para la industria médica, comercialización internacional en tiendas de mascotas, industria ilegal del cuero, pieles y fibras, medicina tradicional, comercio de "buena fortuna", entre otros (SERFOR 2017). A su vez, el SERFOR reportó para el período 2000 - 2015 que el $89 \%$ de las especies intervenidas correspondió a vertebrados donde, los mamíferos representan el 27\% de éstas y además sugirió que las rutas importantes que sigue el tráfico ilegal de fauna silvestre en el Perú involucran la zona nor-oriental, zona centro y zona sur, siendo las dos primeras las que comprometen los departamentos de Amazonas, San Martín, Loreto y Ucayali.

Generar información con escasa base científica o con datos incorrectos puede propiciar errores con repercusiones preocupantes en la gestión de la fauna a nivel nacional. Uno de estos errores podrá ser la presentación de informes técnicos a la Fiscalía Especializada en Materia Ambiental con información errónea, lo cual podría conllevar a un error en la tipificación del delito, dejando sin sanción faltas relacionadas al tráfico de fauna silvestre estipuladas en los Artículos 308 y 309 del Código Penal Peruano. Así por ejemplo, el tráfico ilegal de especies amenazadas extraídas de áreas naturales protegidas por el Estado o de territorios de Comunidades Indígenas, debería sancionarse con una pena privativa de la libertad no menor de cuatro años ni mayor de siete ańos, pero si a causa de un error en la identificación de la especie no llegara a aplicarse esta sanción, se estaría incurriendo en un error de omisión que a largo plazo podría convertirse en una vía permisible al tráfico ilegal. Asimismo, se podría autorizar el manejo o transporte de individuos de una especie amenazada si es confundida con una similar que no se encuentre en alguna categoría de amenaza, esto puede darse en el marco de una autorización, plan de manejo, licencia o contrato. Como en caso contrario, se podría identificar erróneamente una especie no amenazada como una que sí lo es.

\section{Conclusiones}

Aunque consideramos que una guía que permita la fácil identificación de fauna silvestre amenazada es indispensable para 
el adecuado control, fiscalización, supervisión y prevención del tráfico de fauna silvestre en el Perú, también consideramos que esta debe ser realizada de manera interdisciplinaria con la participación de diferentes especialistas con la finalidad de brindar información detallada y confiable, que sirva como herramienta para el público objetivo y, de la misma forma, evitar errores y omisiones como los comentados en esta revisión.

La guía de Allgas et al. (2017) pretende llenar un vacío en la literatura disponible pero, lamentablemente, no logra ser una herramienta adecuada en la identificación de mamíferos silvestres amenazados. Sugerimos que esta guía sea revisada urgentemente para los taxones no tratados en este documento y que la comunidad científica desarrolle este tipo de publicaciones que, aunque no tienen un valor estrictamente científico, son de suma importancia no solamente a nivel ambiental, social y cultural, sino también, sirven como referencia en la toma de decisiones políticas y económicas vinculadas a la gestión de la fauna silvestre en el Perú.

\section{Agradecimientos}

Agradecemos a Fanny M. Cornejo y Fabio Oliveira do Nascimento por los comentarios y sugerencias que enriquecieron este manuscrito.

\section{Literatura citada}

Allgas N., A. Alarcón, N. Shanee, S. Shanee, B. Monteferri \& L. Zari. 2017. Guía de identificación de fauna silvestre, para las autoridades ambientales de Amazonas, San Martín, Loreto y Ucayali. Sociedad Peruana de Derecho Ambiental, Neotropical Primate Conservation Perú. Lima. 128 pp.

Asher R. J. \& K.M. Helgen. 2010. Nomenclature and placental mammal phylogeny. BMC Evolutionary Biology 10(1):102. https://doi.org/10.1186/1471-2148-10-102

Blackwelder R.E. 1967. Taxonomy. A text and reference book. Wiley, New York. 714 pp.

Buckner J.C., J. Lynch Alfaro, A.B. Rylands \& M.E. Alfaro. 2015. Biogeography of the marmosets and tamarins (Callitrichidae). Molecular Phylogenetics and Evolution 82:413-425. https://doi.org/10.1016/j.ympev.2014.04.031

Byrne H., A.B. Rylands, J.C. Carneiro, J.W. Lynch Alfaro, F. Bertuol, M.N.F. da Silva, M.Messias, C.P. Groves, R.A. Mittermeier, I. Farias, T. Hrbek, H. Schneider, I. Sampaio \& J.P. Boubli. 2016. Phylogenetic relationships of the New World titi monkeys (Callicebus): first appraisal of taxonomy based on molecular evidence. Frontiers in Zoology 13:1-26. https:// doi.org/10.1186/s12983-016-0142-4.

Cabrera A. \& J. Yepes. 1960. Mamíferos sudamericanos. Segunda edición, Tomo 1. Ediar, Buenos Aires, 187 pp.

Decreto Supremo No 004-2014-MINAGRI. Decreto Supremo que aprueba la actualización de la lista de clasificación y categorización de las especies amenazadas de fauna silvestre. 08 de abril de 2014. El Peruano, Normas legales: 520497-520504.

Decreto Supremo No 019-2015-MINAGRI. Decreto Supremo que aprueba el Reglamento para la Gestión Forestal. 30 de setiembre de 2015. El Peruano, Normas legales: 562571 562607.

De Oliveira T.G. 1998. Leopardus wiedii. Mammalian Species 579:1-6. https://doi.org/10.2307/3504400

Duplaix N. 1980. Observations on the ecology and behavior of the giant river otter Pteronura brasiliensis in Suriname. Revue d'Ecologie (La Terre et la Vie) 34:495-620.

Gilbert J.A. 2016. Family Dasyproctidae. En: Wilson D.E., T.E. Lacher Jr. \& R.A. Mittermeier, eds. Handbook of Mammals of the World. Volume 6. Lagomorphs and Rodents: Part 1. Lynx Editions, Barcelona.

Groves C.P. 2001. Primate Taxonomy. Smithsonian Institution Press, Washington, DC.
Hernández-Romero P.C., J.A. Guerrero \& C. Valdespino. 2015. Morphological variability of the cranium of Lontra longicaudis (Carnivora: Mustelidae): a morphometric and geographic analysis. Zoological Studies 54(1):50. https:// doi.org/10.1186/s40555-015-0127-6

Hershkovitz P. 1987. The taxonomy of South American sakis, genus Pithecia (Cebidae, Platyrrhini): a preliminary report and critical review with the description of a new species and a new subspecies. American Journal of Primatology 12:387468. https://doi.org/10.1002/ajp.1350120402

Hershkovitz P. 1977. Living New World Monkeys (Platyrrhini) with an Introduction to Primates, volume 1. The Chicago University Press, Chicago.

International Union for Conservation of Nature (IUCN). 2017. The IUCN Red List of Threatened Species. Version 2017 3. <http://www.iucnredlist.org>. Downloaded on 05 December 2017.

Loyola L., L. Escamilo, J. Barrio, J. Benavides \& D.G. Tirira. 2010. Northern pudu Pudu mephistophiles (De Winton 1896). En: J. Barbanti \& S. Gonzales, eds. Neotropical Cervidology: Biology and Medicine of Latin America Deer. Pp. 133-139.

Ley No. 21080. 1975. Aprueban Convención para el Comercio Especies Amenazadas de la Fauna y Flora Silvestre. Actualizado al 13 de marzo de 2012.

Lynch-Alfaro J.W., J.P. Boubli, L.E. Olson, A.D. Fiore, B. Wilson, G.A. Gutiérrez-Espeleta, K.L. Chiou, M. Schulte, S. Neitzel, V. Ross, D. Schwochow, M.T.T. Nguyen, I. Farias, C.H. Janson \& M.E. Alfaro. 2012. Explosive Pleistocene range expansion leads to widespread Amazonian sympatry between robust and gracile capuchin monkeys. Journal of Biogeography 39:272-288. https://doi.org/10.1111/ j.1365-2699.2011.02609.x

Marsh L.K. 2014. A taxonomic revision of the saki monkeys, Pithecia Desmarest, 1804. Neotropical Primates 21:1-163. https:// doi.org/10.1896/044. 021.0101

Matauschek C., C. Roos \& E.W. Heymann. 2011. Mitochondrial phylogeny of tamarins with taxonomic and biogeographic implications for the $\mathrm{S}$. nigricollis species group. American Journal of Physical Anthropology 144:564-574. https:// doi.org/10.1002/ajpa.21445

May C. 2017. (en línea) Transnational Crime and the Developing World. http://www.gfintegrity.org/wp-content/ uploads/2017/03/Transnational_Crime-final.pdf. (Acceso 05/06/2018)

Mittermeier R.A., A.B. Rylands \& D.E. Wilson (eds.). 2013. Handbook of the Mammals of the World: Volume 3. Primates. Lynx Edicions, Barcelona. 953 pp.

Myburgh J. 2011. Crimen transnacional en el mundo en desarrollo. Haken J. Integridad Financiera Global.

Murray J.L. \& G.L. Gardner. 1997. Leopardus pardalis. Mammalian Species 548:1-10. https://doi.org/10.2307/3504082

Novaro A.J. 1997. Pseudalopex culpaeus. Mammalian species 558:18. https://doi.org/10.2307/3504483

Price S.A., O.R. Bininda-Emonds \& J.L. Gittleman. 2005. A complete phylogeny of the whales, dolphins and even-toed hoofed mammals (Cetartiodactyla). Biological Reviews 80:445473. https://doi.org/10.1017/S1464793105006743

Ramírez-Chaves H.E., H.L. Arango-Guerra \& B.D. Patterson. 2014. Mustela africana (Carnivora: Mustelidae). Mammalian Species 917:110-115. https://doi.org/10.1644/917.1

Rheingantz M.L., J.F.S. de Menezes \& B. de Thoisy. 2014. Defining Neotropical otter Lontra longicaudis distribution, conservation priorities and ecological frontiers. Tropical Conservation Science 7(2):214-229. https://doi. org/10.1177/194008291400700204

Rylands A.B., E.W. Heymann, J. Lynch Alfaro, J.C. Buckner, C. Roos, C. Matauschek, J. Boubli, R. Sampaio \& R.A. Mittermeier. 2016. Taxonomic review of the New World tamarins (Primates: Callitrichidae). Zoological journal of the Linnean Society 177:1003-1028. https://doi. org/10.1111/zoj.12386

Servicio Nacional Forestal y de Fauna Silvestre (SERFOR). 2017. Estrategia Nacional para reducir el tráfico ilegal de fauna silvestre, periodo 2017 - 2027. Mavet Impresiones E.I.R.L., Lima. $69 \mathrm{pp}$. 
Sheffield S.R. \& H.H. Thomas. 1997. Mustela frenata. Mammalian Species 570:1-9. https://doi.org/10.1644/0.570.1_

Spaulding M., M.A. O’Leary \& J. Gatesy. 2009. Relationships of Cetacea (Artiodactyla) among mammals: Increased taxon sampling alters interpretations of key fossils and character evolution. PLoS ONE 4(9):e7062. https://doi.org/10.1371/ journal.pone.0007062.

Tirira D.G. 2007. Mamíferos del Ecuador. Guía de campo. Ediciones Murciélago Blanco. Publicación Especial de los Mamíferos del Ecuador 6. Quito.

Tschudi J.J. von. 1844. Untersuchungen über die Fauna Peruana. Scheitlin und Zollikofer. St. Gallen, Suiza. https://doi. org/10.5962/bhl.title.60791_ van Roosmalen M.G.M., T. van Roosmalen \& R.A. Mittermeier. 2002. A taxonomic review of the titi monkeys, genus Callicebus Thomas, 1903, with the description of two new species, Callicebus bernhardi and Callicebus stephennashi, from Brazilian Amazonia. Neotropical Primates 10:1-52.

Wilson D.E. \& R.A. Mittermeier (eds.). 2009. Handbook of the Mammals of the World: Volume 1. Carnivores. Lynx Edicions, Barcelona. 727 pp.

Wilson D.E. \& D.M. Reeder. 2005. Mammals Species of the World, John Hopkins University Press, Baltimore, MD. 2142 pp. 